\title{
Novel Multi-Step Recursive Sampling Strategy for Particle Filtering in object tracking
}

\author{
Zhang Chen, and Wan-Chi Siu, Fellow, IEEE \\ Centre for Signal Processing, Department of Electronic and Information Engineering \\ The Hong Kong Polytechnic University, Kowloon, Hong Kong \\ Email: ericcz0210@gmail.com, enwcsiu@polyu.edu.hk
}

\begin{abstract}
In this paper, we propose a new sampling strategy for particle filtering in object tracking. Improper sampling can bring in heavy computation for achieving acceptable tracking results in particle filter. We propose a multi-step recursive sampling method to replace the direct importance sampling. This relies on the feedback from the resampling procedure. New particles are sampled recursively from the existing particles with high weights. After the iterations, particles become densely populated, and this approach contributes significantly to achieve accurate position estimation. Experimental results indicate that the method reduces computation substantially and it also preserves good tracking results.
\end{abstract}

Keywords-object tracking; particle filtering; multi-step sampling; resampling, recursive sampling

\section{INTRODUCTION}

Video Tracking is an important part of object monitoring and surveillance. It aims at locating object position in the video sequence, which can be done using particle filtering method. Typically, the particle filter (PF) [1] follows the sequential Monte-Carlo method to form a discrete expression of posterior pdf (probability density distribution) for states given known observations. No doubt, the PF has attracted attention of many researchers. The SIR (sequential importance resampling) Particle Filter $[1,2,3]$, also known as Bootstrap filter, is the most widely used particle filter. However, a large amount of particles should be sampled, w`hich results in loss of simplicity and efficiency, are exploited in the likelihood to compute the weights as discrete coefficients of the posterior density. Some recent works $[2,4,5]$ succeeded in improving the proposal distribution which is closer to the prior transition density. However, the number of particles is not reduced. Namely, the computation complexity is still high. Thus some approaches intend to reduce the complexity while still succeed in tracking. The hybrid particle filter with mean shift (HY) [6] tracker is an example that uses mean shift to refine the particles. On the other hand, Such as multistage sampling in [7], MCMC(Markov Chain Monte Carlo) PF in [8] and Annealed $\mathrm{PF}$ in $[9,10]$, the sampling does not involve any direct filtering operation or refinement of individual particles, but is done step by step according to the feedback of existing samples.

In this paper, we propose a new multi-step recursive sampling in a resampling Algorithm, which aims at finding more efficient and fewer particles while preserving the tracking results robustly. In our approach, selected particles at each step are involved into the likelihood computation and resampling procedure. The residual particles with top weights are then extracted for the next sampling step. Thus some low-weight particles are filtered, and only the more possible ones are activated for further sampling. Recursively, the optimal object location can be confirmed by the promising particles.

The rest of the paper is organized as follows. Section II gives a description of our new sampling strategy. The realization of this approach is given in section III. Simulation and experimental results are reported in Section IV. Finally, in Section V, we conclude the paper.

\section{MULTI-STEP SAMPLING}

\section{A. Background Theory}

Let us define $x_{k}$ as the target state at time $\mathrm{k}$ and $z_{1: k}$ denotes a set of past $\mathrm{k}$ observations. $\mathrm{x}_{0}$ is the prior knowledge, $\mathrm{z}_{k}$ is the observation at time $\mathrm{k}$, whereas $\mathrm{z}_{0}$ is meaningless. In the particle filter, the posterior probability density function ( $\mathrm{pdf}) \mathrm{p}\left(\mathrm{x}_{k} \mid \mathrm{z}_{1: k}\right)$ is characterized by a set of $\mathrm{N}$ weighted samples or particles, namely, $\left\{x_{k}^{i}, \omega_{k}^{i}\right\}_{i=l}^{N}$, where i specifies particle index, $\omega_{k}^{i}$ is the weight of particle $x_{k}^{i}$ and is usually normalized. Then, practically, the posterior density [2] at state $\mathrm{k}$ can be approximated as

$$
p\left(x_{k} \mid z_{1: k}\right) \approx \sum_{i=1}^{N} \omega_{k}^{i} \delta\left(x_{k}-x_{k}^{i}\right) .
$$

In the Hidden Markov Model, the expectation of particles is usually the estimate in a uni-modal weight distribution. If the sampled particles are mainly chosen from the high-likelihood region, where the particle density is high around the global maximum, the expected estimate will closely approximate to the peak point with the real global maximum in weight.

This inspires us to propose the idea of multi-step recursive sampling for more top-weight particles. Therefore, we will perform the sampling in chain actions. It means drawing particles in a path of steps, which is shown in Fig.1 (a) and (b). A state set contains all the possible candidates in particles. As shown in Fig.1 (a), it is the standard sampling process between steps. New particles at next step are generated based on the existing ones and a Gaussian sampling function drawn in dotline curve. From the likelihoods in terms of density $p\left(z_{k} \mid x_{k}\right)$, we can observe that a new particle is more probable to stay in the

978-1-4673-2197-6/12/\$31.00 @2012 IEEE 
high-likelihood region if its referenced particle (blue blob) is also in it.

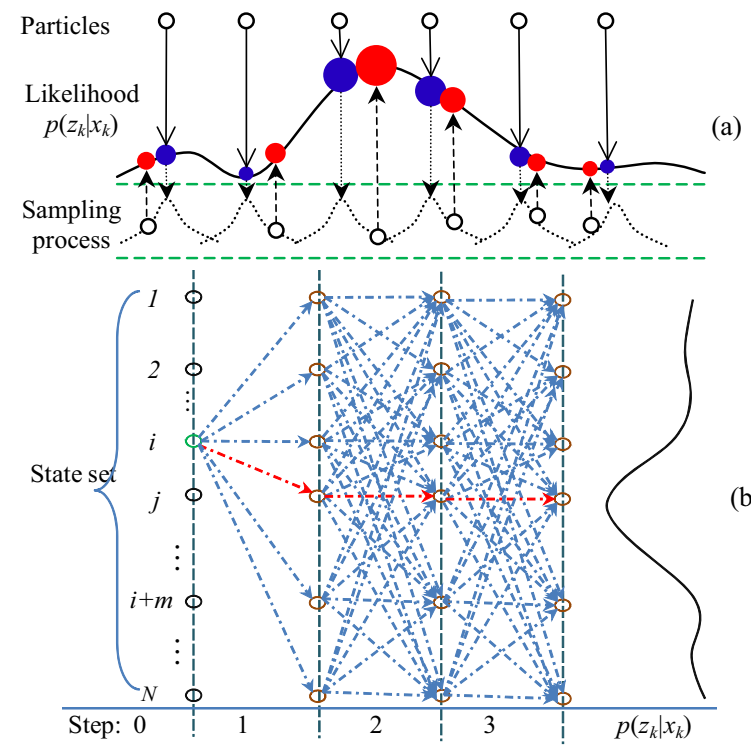

Figure 1. simulation results of recursive sampling: (a) example of sampling process between two steps, (b) the most prominent chain path given the likelihood density.

After several steps, these particles at every step can be linked together to form a possible path which is in the form of a particle chain. In Fig.1 (b), the candidates (from 1 to $\mathrm{N}$ ) in the state set are connected to candidates of the next steps, with the blue arrow lines. Each candidate also owns its likelihood from the density $p\left(z_{k} \mid x_{k}\right)$. Overall, there are huge amount of possible routes in this example. Objectively, the most prominent chain with all leading particles is the wanted set of particles, such as the red arrows in Fig.1 (b). Unlike other less efficient algorithms, we have fewer samples in demand.

\section{B. Derivation of the sampling strategy}

Since, after resampling and predictive transitions, the existing particles are considered as the starts of the chains. Subsequently, the estimation process relies on the newly proposed particles in the chains. Hence, we define a new set $\left\{x_{k, 0: S}^{i}\right\}$ as the $\mathrm{i}^{\text {th }}$ particle chain which is a list of consecutive particle records $x_{k, s}^{i}$ from steps 0 to $\mathrm{S}$, where $\mathrm{S}$ is the final step order of the chain, $s$ is one instant of steps in $[0, S]$, and $i$ labels the particle chain which is equal to the index of a starting particle among all particles in the initial stage. Let us also denote $\omega_{k, 0: S}^{i}$ as the weight of the particular particle chain $x_{k, 0: S}^{i}$. As $\omega_{k, 0: s}^{i}$ is related to discrete particle weights, when its value is high, the chain is better in particle sampling. Hence, our objective is to let each chain obtain weights with values as high as possible. Particles with the promising route are most possibly staying in the high-likelihood area. Then convert eqn (1) into

$$
p\left(x_{k} \mid z_{1: k}\right) \approx \sum_{j=1}^{n} \sum_{s=0}^{S} \widetilde{\omega}_{k, s}^{j} \delta\left(x_{k}-\widetilde{x}_{k, s}^{j}\right)
$$

where $\left\{\widetilde{x}_{k, 0: S}^{j}, \widetilde{\omega}_{k, 0: S}^{j}\right\}=\underset{j=1 \ldots n}{\arg \max }\left(\omega_{k, 0: S}^{i}\right)$,

i.e. $\tilde{x}_{k, 0: S}^{j}$ is the chain of particles with the $j^{\text {th }}$ largest weight $\widetilde{\omega}_{k, 0: s}^{j}$ at time k. $n$ is the chosen number of top-weight chains. By the way, the largest weight indicates that particles in this chain will reach the high region as soon as possible and the weights of individual particles will be high as well. Then, the weight $\omega_{k, 0: S}^{i}$ can be expressed according to the importance sampling, i.e.

$$
\omega_{k, 0: S}^{i} \propto \frac{p\left(x_{k, 0: S}^{i} \mid z_{1: k}\right)}{q\left(x_{k, 0: S}^{i} \mid z_{1: k}\right)} .
$$

where $p\left(x_{k, 0: S}^{i} \mid z_{1: k}\right)$ is the posterior pdf output of the $i^{\text {th }}$ particular chain, and $q\left(x_{k, 0: S}^{i} \mid z_{1: k}\right)$ is the importance sampling strategy in pdf.

For a further deviation from model equations in $[1,2]$, the chain weight is updated by

$\omega_{k, 0: S}^{i} \propto \frac{p\left(z_{k} \mid x_{k, 1: S}^{i}\right)\left\{\prod_{s=0}^{S-1} p\left(x_{k, s+1}^{i} \mid x_{k, s}^{i}\right)\right\}}{\left\{\prod_{s=0}^{S-1} q\left(x_{k, s+1}^{i} \mid x_{k, s}^{i}\right)\right\}} \omega_{k, 0}^{i}$

As likelihood of particle chain $p\left(z_{k} \mid x_{k, 0: S}^{i}\right)$ is given by the multiplications of the all individual likelihood outputs $p\left(z_{k} \mid x_{k, s}^{i}\right)$, we have

$$
p\left(z_{k} \mid x_{k, 1: S}^{i}\right)=p\left(z_{k} \mid x_{k, 1}^{i}\right) p\left(z_{k} \mid x_{k, 2}^{i}\right) \ldots p\left(z_{k} \mid x_{k, S}^{i}\right) .
$$

Among s steps, $p\left(x_{k, s+1}^{i} \mid x_{k, s}^{i}\right)=q_{s}\left(x_{k, s+1}^{i} \mid x_{k, s}^{i}\right)$, namely, the proposed distribution is equal to the prior transition distribution. And $\omega_{k, 0}^{i}$ is the weight of the beginning $(\mathrm{s}=0)$ particle in the $i^{\text {th }}$ chain after predictive transition.

Consequently, the weight of particle chain $\omega_{k, 0: S}^{i}$ can be simplified as the multiplications of the likelihood at each step $p\left(z_{k} \mid x_{k, s}^{i}\right)$ and the initial weight $\omega_{k, 0}^{i}$ before the multi-step sampling.

$$
\begin{aligned}
& \omega_{k, 0: S}^{i} \propto \prod_{s=1}^{S} p\left(z_{k} \mid x_{k, s}^{i}\right) \omega_{k, 0}^{i} \\
& \omega_{k, 0}^{i} \propto p\left(z_{k} \mid x_{k, 0}^{i}\right) \omega_{k-1}^{j}
\end{aligned}
$$

where $\omega_{k-1}^{j}$ is the weight of the residual particle after resampling at the last stage .

Recall the Vertibi algorithm, the most probable list of instances is the combination of those which have maximized the probability at corresponding steps. As the maximum of $\omega_{k, 0: S}^{i}$ is essential for an efficient sampling, through the Vertibi algorithm, the expression in eqn (6) is converted into maximizing weight of the chain at each sampling step, i.e.

$\omega_{k, 0: s}^{i}=\max _{x_{k, s}^{i}}\left[p\left(z_{k} \mid x_{k, s}^{i}\right) \omega_{k, 0: s-1}^{i}\right]$, when $\mathrm{s}>0$ 
In other words, with a considerable number of samples, if we can obtain the particle with the highest likelihood at each step from eqn (7), the chain of particles can finally converge to the area holding the optimal candidate. The mathematical derivation has thus proved that the multi-steps sampling is effective to estimation in the discrete form. Because the optimal estimate is considered to have the highest product of weights, it is certain that a proper particle chain should have the highest possibility. Also this particular chain must be the fastest route (fewest steps) towards the peak area (the most probable state) of the estimate. Hence, our objective is to focus on finding the routes of particles with the highest weight.

\section{REALIZATION}

In general, the recursive sampling algorithm (RS) is combined with the modified resampling strategy (RSMPL). We have used both to formulate our new particle filtering algorithm. Thus this Multi-Step Recursive Sampling in Resampling (MSRSIR) Algorithm can be illustrated in Table I.

TABLE I. ALGORITHM OF MSRSIR

$\left[\left\{x_{k}^{i *}, \omega_{k}^{i *}\right\}_{i=1}^{N_{k}},\left\{x_{k}^{j}, \omega_{k}^{j}, t^{j}\right\}_{j=1}^{N^{\prime}}\right]=\operatorname{RSIRPF}\left[\left\{x_{k-1}^{i}, \omega_{k-1}^{i}, t^{i}\right\}_{i=1}^{N^{\prime}}, Z_{k}\right]$

Initialization: sampling step $\mathrm{s}=0$

Sampling $N_{0}$ particles by prediction and assumption $\left(N_{0}=\right.$ $\left.N_{k, 1}\right)$

$$
\left[\left\{x_{k, 0}^{j}, \omega_{k, 0}^{j}\right\}_{j=1}^{N_{k, 0}}\right]=\operatorname{RS}\left[\left\{x_{k-1}^{i}, \omega_{k-1}^{i}, t^{i}\right\}_{i=1}^{N^{\prime}}, 0\right]
$$

- Assign the particle a weight $\omega_{k, 0}^{j} \propto p\left(z_{k} \mid x_{k, 0}^{j}\right) \omega_{k-1}^{j}$

Normalize those weights with sum equal to 1 .

Resample those $N_{k, 1}$ particles

$$
\left[\left\{x_{k, 0}^{j}, \omega_{k, 0}^{j}, t_{1}^{j}\right\}_{j=1}^{N_{k, 1}^{\prime}}\right]=\operatorname{RSMPL}\left[\left\{x_{k, 0}^{i}, \omega_{k, 0}^{i}\right\}_{i=1}^{N_{k, 0}}\right]
$$

For $\mathrm{s}=1: S$ (Maximum)

- Sampling according to the resampled results

$$
\left\{x_{k, s}^{j}, \omega_{k, s}^{j}\right\}_{j=N_{k, s-1}}^{N_{k, s}}=\operatorname{RS}\left[\left\{x_{k, s-1}^{i}, \omega_{k, s-1}^{i}, t_{s-1}^{i}\right\}_{i=1}^{N_{k, s-1}^{k}}, N_{k, s-1}\right]
$$

- Assign the particle a weight using likelihood

- Normalize those weights with sum equal to 1

- Resampling these $N_{k, s}$ particles

$$
\left[\left\{x_{k, s}^{j}, \omega_{k, s}^{j}, t_{s}^{j}\right\}_{j=1}^{N_{k, s}^{\prime}}\right]=\operatorname{RSMPL}\left[\left\{x_{k, s}^{i}, \omega_{k, s}^{i}\right\}_{i=1}^{N_{k, s}}\right]
$$

- If $\mathrm{N}_{\mathrm{k}, \mathrm{s}}>\mathrm{N}$ ( $\mathrm{N}$ is the stopping threshold of particle No.)

- $\mathrm{N}_{\mathrm{k}}=\mathrm{N}_{\mathrm{k}, \mathrm{s}}$, and $\left\{x_{k}^{j}, \omega_{k}^{j}, t^{j}\right\}_{j=1}^{N^{\prime}}=\left\{x_{k, s}^{j}, \omega_{k, s}^{j}, t_{s}^{j}\right\}_{j=1}^{N_{k, s}^{\prime}}$

- Stop iterations.

\section{End FOR}

Collect all sampled particles in $\left\{x_{k}^{i *}, \omega_{k}^{i *}\right\}_{i=1}^{N_{k, S}}\left(\omega^{i^{*}}{ }_{k}\right.$ is kept un-normalized.)

Calculate the total weight of all sampled particles

Normalize each particle.

Compute the expectation using eqn (2)

As for systematic resampling, we can collect the times of replication for particles. $\left[\left\{x_{k}^{j^{*}}, \omega_{k}^{j}, t^{j}\right\}_{j=1}^{N^{\prime}}\right]=$ RSMPL $\left[\left\{x_{k}^{i}, \omega_{k}^{i}\right\}_{i=1}^{N}\right]$, where $\mathrm{t}$ denotes the times of replication for $i^{\text {th }}$ particle and $\mathrm{j}$ is the index of the residual particles. After resampling, as shown in fig.1 (a), the recursive sampling (RS) priority depends on the repetition of residual particles. The most repeated particle owns the highest priority than others. It will be arranged to proceed sampling firstly. The largest portion of particles will be allocated to samples around it. Intuitively, we sample a few particles as pioneers. Then resample them in terms of the density distribution. The next sampling step is performed based on the coarse density distribution. According to the repetitions (t) of the residual particles, new samples are picked around them with the corresponding priority. The input set $\left\{x_{k, s-1}^{i}, \omega_{k, s-1}^{i}, t_{s-1}^{i}\right\}_{i=1}^{N_{k, s-1}^{\prime}}$ is the resampled particle set from the previous step. $\mathrm{N}_{k, s-1}$ denotes the number of already sampled particles before step s. An upper-bounded particle number $\left(N_{s}\right)$ is chosen at each step for regulating the sample size. Additionally, we set up a decreasing threshold of repetition for more possible routes.

\section{EXPERIMENTS}

\section{A. Subjective evaluation}

In our experiments, some parameters were pre-defined empirically as shown in Table II. The tracking results are illustrated in the Fig. 2.

TABLE II. PARAMETERS OF FOUR APPROACHES IN REALIZATION

\begin{tabular}{|l|l|l|l|l|}
\hline Method & $\begin{array}{l}\text { Particle } \\
\text { No. (N) }\end{array}$ & $\begin{array}{l}\text { Sampling } \\
\text { pattern }\end{array}$ & Steps & $\begin{array}{l}\text { sample } \\
\text { refinement }\end{array}$ \\
\hline SIR PF [3] & 200 & $\begin{array}{l}\mathrm{x} \sim \mathrm{N}(0,10) \\
\mathrm{y} \sim \mathrm{N}(0,10)\end{array}$ & 1 & No \\
\hline APF [9,10] & 14 & $\begin{array}{l}\mathrm{x} \sim \mathrm{N}\left(0,10 \times 0.8^{\mathrm{s}}\right) \\
\mathrm{y} \sim \mathrm{N}\left(0,10 \times 0.8^{\mathrm{s}}\right)\end{array}$ & 5 & replacement \\
\hline HY [6] & 15 & $\begin{array}{l}\mathrm{x} \sim \mathrm{N}(0,10) \\
\mathrm{y} \sim \mathrm{N}(0,10)\end{array}$ & 1 & Mean shift \\
\hline $\begin{array}{l}\text { Our } \\
\text { Method }\end{array}$ & $\begin{array}{l}\mathrm{N}_{0}=20, \\
\mathrm{~N}_{\mathrm{s}}=15, \\
\mathrm{~N}=50\end{array}$ & $\begin{array}{l}\mathrm{x} \sim \mathrm{N}\left(0,10 \times 0.8^{\mathrm{s}}\right) \\
\mathrm{y} \sim \mathrm{N}\left(0,10 \times 0.8^{\mathrm{s}}\right)\end{array}$ & $3-4$ & No \\
\hline
\end{tabular}

The black box represents the current estimates [2, 11]. The dots in the frame are the current distribution of particles. For example, in Fig.2(a), after the SIR particle filtering, most particles (blue dots) are spreading away from the potential area with little contribution to the estimate. The rest particles with weights higher than the average are drawn in red dots. Other three approaches which make use of fewer particles are as shown in Table II. In the soccer sequence, the ball was stable with nearly no motion in the beginning frames (1st 10th frame). Then, in frame 18, the ball was kicked with a rapid acceleration, and was later occluded by the legs. Note that, when the ball is still, the performance of Annealed PF (b) and HY (c) is worse than other two methods. Due to not enough density, they met the drift problem by fluctuating around the real target. However, our method (by collecting all particles together) does not have such problem. After the ball kicked, the ball was accelerated to fast velocity in a short time. Also, since the feet are similar to the ball in color, the generated multi-modal likelihood map increases the difficulty to capture the target. Hence, the processes of sampling in steps and particle refinement have inevitably to search more effective particles in the right region. Observing from the results, the three methods except SIR PF (a) are positively reacting to that. As the occlusion shows up, though some 
particles find the correct target region as in SIR PF (a) and Annealed PF (b), the estimated location does not match well. However, the mean-shift procedure cannot do well on refinement, the HY approach even lost tracking of the soccer ball as shown in Fig.2 (c). Nevertheless, the performance of the proposed method is still the best among all algorithms by observing the target carefully.

a)

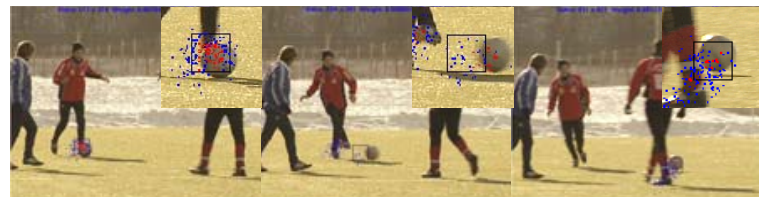

b)

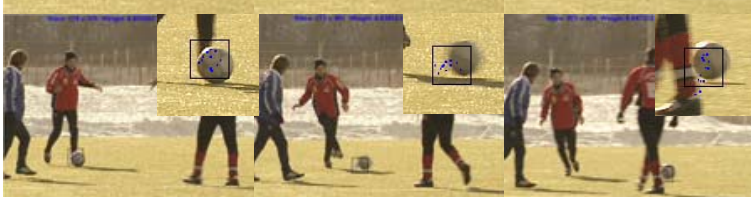

c)

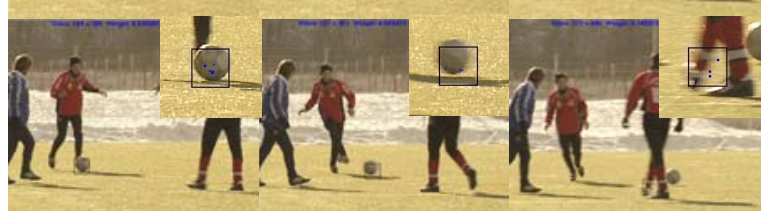

d)

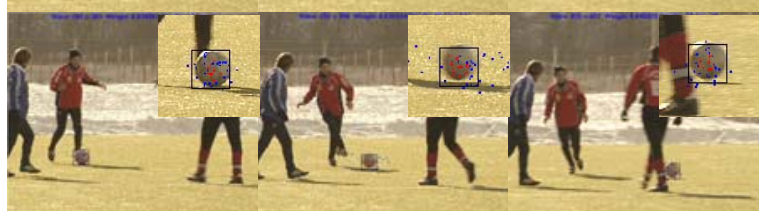

Figure 2. comparison of the subjective test in the soccer sequence, a) the SIRPF, b) APF, c) HY and d) our proposed

\section{B. Computation comparison}

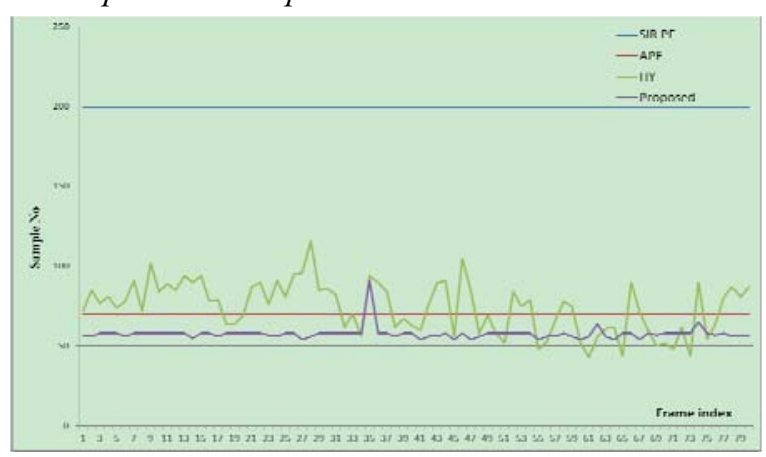

Figure 3. Comparison of the computation cost in "particles" using these four methods

In Fig. 3, The SIR PF needs 200 particles for acceptable results. If enough particles and proper searching region are provided, the final estimate will converge to the optimal one. However, it is a waste of computation in calculating some redundant particles mentioned before. Hence, the Annealed PF and HY were proposed to save computation by proposing fewer particles. The Annealed PF samples particles in layers and each layer contains the same particle number. In the soccer video sequence, totally, 70 particles were settled. For HY, the computation in likelihood is also used in mean-shift iterations.
For the simplification of comparison, it can be regarded as processing of one particle. From fig.3, the number particle computation (real particles $+\mathrm{MS}$ iteration) for the $\mathrm{HY}$ algorithm varies from 48 to 110 , depending upon MS. In experiment, approximately, the average count is 72 in the terms of "particle" computation. Besides, ours has succeeded in saving computation. On average, 56 particles were sampled, which is the fewest number of particles among all algorithms. Hence, the computation cost is the lowest comparing with other three methods. Meanwhile, the tracking result is the most successful among all the four approaches given such a limitation on computation.

\section{CONCLUSION}

This paper presented a new adaptive sampling approach for particle filtering. Rather than sampling all particles in one step, we proposed a multi-step recursive sampling method, which refers to resampling strategy. It is designed to look for highweight particles as many as possible in steps. Thus the sampled particles are more efficient in estimation, which is closer to the highly probable area. Experimental results have verified the effectiveness of the proposed method in reducing computation and improving robustness of our algorithm.

\section{REFERENCES}

[1] Doucet A., Nando de Freitas and Gordon N.J., "Sequential Monte Carlo methods in practice", New York: Springer-Verlag, pp146-152, 2001.

[2] Arulampalam M.S., Maskell S., Gordon N., Clapp T., "A tutorial on particle filters for online nonlinear/non-Gaussian Bayesian tracking", IEEE Transactions on Signal Proce., vol. 50, no.2, pp. 174-188, 2002.

[3] Nummiaro K., Koller-Meier E. and Gool, L. V., "A Color-Based Particle Filter", Proceedings, 1st International Workshop on Generative-ModelBased Vision (in conjunction with ECCV02), Denmark, pp. 53-60, Jun 2002.

[4] Merwe, R., Doucet, A., Freitas, N., and Wan, E., "The unscented particle filter", Technical Report CUELYF-INFEh'GAR 380, Cambridge University Engineering Depart., Aug. 2000.

[5] Pan, P. and Schonfeld, D.,"Dynamic Proposal Variance and Optimal Particle Allocation in Particle Filtering for Video Tracking", IEEE Trans. on CSVT, vol.18, no.9, pp.1268-1279, Sept. 2008

[6] Bohyung Han, Ying Zhu, Comaniciu, D. and Davis, L.S., "Visual Tracking by Continuous Density Propagation in Sequential Bayesian Filtering Framework", IEEE Trans. PAMI, vol.31, no.5, pp.919-930, May 2009.

[7] Khan, Z., Balch, T., and Dellaert, F., "MCMC-Based Particle Filtering for Tracking a Variable Number of Interacting Targets," IEEE Trans. PAMI, Vol. 27, No. 11, pp. 1805-1819, Nov. 2005.

[8] Maggio, E., Cavallaro, A., "Hybrid Particle Filter and Mean Shift tracker with adaptive transition model", Proceedings, ICASSP'05, Philadelphia, PA, USA, vol.2, pp. 221- 224, March, 2005.

[9] J. Deutscher, A. Blake, and I. Reid, "Articulated body motion capture by annealed particle filtering”, Proceedings, CVPR'00, Hilton Head, SC, pp. 126-133, 2000.

[10] Fontmarty, M., Lerasle, F. and Danes, P., "Data fusion within a modified annealed particle filter dedicated to human motion capture", Proceedings, IEEE/RSJ International Conference on Intelligent Robots and Systems, San Diego, CA, USA, pp.3391-3396, 2007.

[11] Kin-Yi Yam, Wan-Chi Siu, Ngai-Fong Law, and Chok-Ki Chan, "Effective bi-directional people flow counting for real time surveillance system", Proceedings, ICCE'11, Las Vegas, USA, pp. 863-864, 9-12 Jan.2011. 